\title{
Impact of long-term exposure to PM2.5 and temperature on coronavirus disease mortality: observed trends in France
}

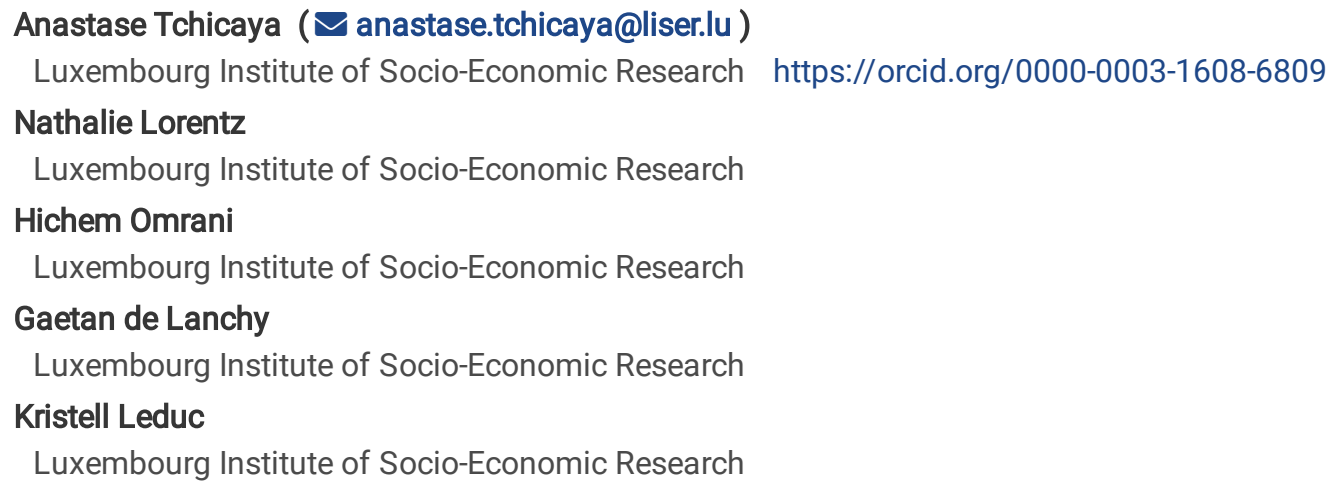




\section{Abstract}

Background The outbreak of coronavirus disease (COVID-19) began in Wuhan, China in December 2019 and was declared a global pandemic on 11 March 2020. This study aimed to assess the effects of temperature and long-term exposure to air pollution on the COVID-19 mortality rate at the sub-national level in France.

Methods This cross-sectional study considered different periods of the COVID-19 pandemic from May to December 2020 . It included 96 departments (or NUTS 3) in mainland France. Data on long-term exposure to particulate matter $\left(\mathrm{PM}_{2.5}\right)$, annual mean temperature, health services, health risk, and socio-spatial factors were used as covariates in negative binomial regression analysis to assess their influence on the COVID-19 mortality rate. All data were obtained from open-access sources.

Results The cumulative COVID-19 mortality rate by department increased during the study period in metropolitan France-from 19.8/100,000 inhabitants (standard deviation (SD): 20.1) on 1 May 2020, to 65.4/100,000 inhabitants (SD: 39.4) on 31 December 2020. The rate was the highest in the departments where the annual average of long-term exposure to $\mathrm{PM}_{2.5}$ was high. The negative binomial regression models showed that a $1 \mu \mathrm{g} / \mathrm{m}^{3}$ increase in the annual average $\mathrm{PM}_{2.5}$ concentration was associated with a statistically significant increase in the COVID-19 mortality rate, corresponding to $24.4 \%, 25.8 \%, 26.4 \%, 26.7 \%, 27.1 \%, 25.8 \%$, and $15.1 \%$ in May, June, July, August, September, October, and November, respectively. This association was no longer significant on 1 and 31 December 2020. The association between temperature and the COVID-19 mortality rate was only significant on 1 November, 1 December, and 31 December 2020. An increase of $1^{\circ} \mathrm{C}$ in the average temperature was associated with a decrease in the COVID-19-mortality rate, corresponding to $9.7 \%, 13.3 \%$, and $14.5 \%$ on 1 November, 1 December, and 31 December 2020 , respectively.

Conclusion This study found significant associations between the COVID-19 mortality rate and long-term exposure to air pollution and temperature. However, these associations tended to decrease with the persistence of the pandemic and massive spread of the disease across the entire country.

\section{Background}

The outbreak of coronavirus disease (COVID-19) began in Wuhan, China in December 2019, and quickly spread worldwide. COVID-19 is a respiratory infection caused by severe acute respiratory syndrome coronavirus 2 (SARS-CoV-2). The World Health Organization (WHO) declared a state of global health emergency on 31 January 2020 [1] and the state of a pandemic on 11 March 2020. Several epidemiological studies have shown that air pollution increases the incidence of a wide range of diseases, mainly respiratory and cardiovascular diseases [2-6]. Exposure to air pollution is associated with vulnerability, including low socioeconomic status (SES), highrisk occupation, and pre-existing health problems $[3,6,7]$. People with low SES are more likely to work outdoors or in places with air pollution or extreme temperatures [5]. In contrast, very high-income groups tend to work indoors, which reduces their exposure [3,5].

Furthermore, people with pre-existing health problems tend to be significantly affected by air pollution. For example, heart attack survivors are more likely to be readmitted to hospitals and have a very high mortality rate if they are exposed to long-term air pollution [6].

The association between long-term exposure to $\mathrm{PM}_{2.5}$ and mortality is well documented [2, 4, 8-12], with chronic exposure to $\mathrm{PM}_{2.5}$ most strongly associated with mortality attributable to ischemic heart disease, arrhythmia, heart failure, and cardiac arrest [2].

Most studies conducted since the beginning of the COVID-19 pandemic have shown that environmental factors such as air pollution and ambient temperature can be considered crucial mediators in COVID-19 spread and mortality [13-20]. An analysis of the spatial or geographic distribution of severe infections and deaths from COVID-19 highlighted that populations living in areas with high levels of air pollution were more likely to develop severe COVID-19 or die than other populations. The mechanisms by which these two environmental factors interact with COVID-19 are not well established. For example, Wang et al. [14] considered that $\mathrm{PM}_{2.5}$ could facilitate SARS-CoV-2 infection through overregulation of angiotensin-converting enzyme 2 (ACE2).

In France, in 2015 , the annual mean $\mathrm{PM}_{2.5}$ concentration level was $11.9 \mu \mathrm{g} / \mathrm{m}^{3}$ and was responsible for 35,800 premature deaths and 624 years of life lost (7). The average concentration level of $\mathrm{PM}_{2.5}$ slightly above the $10 \mu \mathrm{g} / \mathrm{m}^{3}$ limit set by the WHO masks the spatial disparities in $\mathrm{PM}_{2.5}$ exposure and its impact on the COVID -19 spread and mortality. However, further investigations are needed.

As of 31 December 2020, France declared 44,456 cumulative deaths in hospitals due to COVID-19 (Ministry of Health/Directorate General of Health 2020). The spatial distribution of COVID-19 mortality was unequal across departments, but the determinants of unevenness were unknown. 
Therefore, the present study aimed to assess the effects of long-term exposure to both air pollution and temperature on the COVID-19 mortality rate in mainland France, at different times of the evolution of COVID-19 pandemic. To the best of our knowledge, this study is the first to assess the combined associations of two environmental factors with COVID-19 mortality rate measured over a relatively large period at the sub-national level in France or in Europe. Further, this study measured the risk of COVID-19 mortality with regard to the PM 2.5 concentration level by quartile to highlight the impact of inequalities in the long-term exposure to $\mathrm{PM}_{2.5}$ and its disparities in COVID-19 mortality rates observable at the sub-national level.

\section{Materials And Methods}

\section{Design}

This study cross-sectional analysis used data on COVID-19 mortality, environmental factors (PM2.5 and temperature), and socio-spatial factors measured at the level of the departments (NUTS 3). The cross-sectional analysis was performed on different dates during the pandemic (from 1 May to 31 December 2020) across 96 departments of mainland France. Two epidemic waves were observed during the study period, with the second wave emerging from September (Supplementary Material Fig. 1).

From this perspective, only the cumulative number of deaths changed, and all other independent variables characterising the different departments remained unchanged. One of the objectives was to understand the extent to which pre-existing conditions before the COVID19 outbreak could play a significant role in the COVID-19 spread and severity observed in the country despite implementation of various control measures at the national level (such as lockdown, physical and social distancing, curfews, teleworking, travel restrictions, and wearing masks).

\section{Data sources and data}

Several data sources were used in this study, and all the variables used are shown in Table 1 (the full dataset is publicly accessible) [21]. Cumulative data on COVID-19 deaths in the hospitals were obtained from the French Ministry of Health statistics.

Data on environmental factors, including exposure to $\mathrm{PM}_{2.5}$ and air temperature, were obtained from the Global Ozone Monitoring Experiment and the World Climate, respectively (Table 1). Air pollution (i.e. $\mathrm{PM}_{2.5}$ ) data were downloaded from the NASA-Socioeconomic Data and Applications Centre. Temperature data were obtained from WorldClim-Version2. Initially, both variables had a resolution of $1 \mathrm{~km}$ $\times 1 \mathrm{~km}$ (or $1 \mathrm{~km}^{2}$ ), but later they were averaged and aggregated (using a mean operator) to obtain long-term exposure to air pollution (1999-2016) and temperature (1996-2012) at the departmental level (NUTS 3) in mainland France.

Table 1: Variables, definitions and data sources 


\begin{tabular}{|c|c|c|c|c|}
\hline Variables & Definition & Data sources & $\begin{array}{l}\text { Data } \\
\text { year }\end{array}$ & $\begin{array}{c}\text { Level } \\
\text { considered }\end{array}$ \\
\hline $\begin{array}{l}\text { COVID-19 death } \\
\text { rate in hospitals }\end{array}$ & $\begin{array}{l}\text { Number of cumulated COVID- } \\
19 \text { deaths in hospitals } \\
\text { expressed per } 100000 \text { people }\end{array}$ & $\begin{array}{l}\text { Ministry of Health } \\
\text { https://www.data.gouv.fr/fr/datasets/donnees- } \\
\text { hospitalieres-relatives-a-lepidemie-de-covid- } \\
\text { 19/ }\end{array}$ & 2020 & Department \\
\hline \multicolumn{5}{|c|}{ Availability of healthcare services } \\
\hline $\begin{array}{l}\text { Number of } \\
\text { resuscitation } \\
\text { beds }\end{array}$ & $\begin{array}{l}\text { Number of resuscitation beds } \\
\text { expressed per } 100000 \text { people }\end{array}$ & $\begin{array}{l}\text { Ministry of Health } \\
\text { https://drees.solidarites-sante.gouv.fr }\end{array}$ & 2018 & Department \\
\hline $\begin{array}{l}\text { Number of } \\
\text { intensive care } \\
\text { beds }\end{array}$ & $\begin{array}{l}\text { Number of intensive care beds } \\
\text { expressed per } 100000 \text { people }\end{array}$ & $\begin{array}{l}\text { Ministry of Health } \\
\text { https://drees.solidarites-sante.gouv.fr }\end{array}$ & 2018 & Department \\
\hline $\begin{array}{l}\text { Physicians } \\
\text { density }\end{array}$ & $\begin{array}{l}\text { Number of physicians divided } \\
\text { by the department area }\end{array}$ & $\begin{array}{l}\text { Ministry of Health } \\
\text { https://drees.solidarites-sante.gouv.fr }\end{array}$ & 2018 & Department \\
\hline \multicolumn{5}{|c|}{ Socio-spatial factors } \\
\hline $\begin{array}{l}\text { \%People aged } \\
60+\end{array}$ & $\begin{array}{l}\text { Proportion of the population } \\
60 \text { years of age or older }\end{array}$ & Eurostat & 2019 & Department \\
\hline \%Males & $\begin{array}{l}\text { Proportion of male sex in } \\
\text { population }\end{array}$ & Eurostat & 2019 & Department \\
\hline \%Unemployment & $\begin{array}{l}\text { Proportion of adults } \\
\text { unemployed adults }\end{array}$ & Eurostat & 2019 & Department \\
\hline $\begin{array}{l}\text { \%Urban } \\
\text { population }\end{array}$ & $\begin{array}{l}\text { Proportion of people living in } \\
\text { the greater urban areas (per } \\
\text { cent). }\end{array}$ & $\begin{array}{l}\text { FNORS/STATISS 2019, version of May 18, } \\
2020\end{array}$ & 2016 & Department \\
\hline Rate of poverty & $\begin{array}{l}\text { Rate of monetary poverty (per } \\
\text { cent) }\end{array}$ & FNORS & 2016 & Department \\
\hline $\begin{array}{l}\text { Population } \\
\text { density }\end{array}$ & $\begin{array}{l}\text { Number of people by square } \\
\text { kilometer }\end{array}$ & Eurostat & 2019 & Department \\
\hline Population size & $\begin{array}{l}\text { Size of population for each } \\
\text { department (by thousand) }\end{array}$ & Eurostat & 2019 & Department \\
\hline \multicolumn{5}{|l|}{ Health risk factors } \\
\hline $\begin{array}{l}\text { Standardized } \\
\text { Prevalence rate of } \\
\text { Diabetes }\end{array}$ & $\begin{array}{l}\text { Standardized prevalence rate } \\
\text { of pharmacologically treated } \\
\text { diabetes (all types) (\%). }\end{array}$ & $\begin{array}{l}\text { https://www.santepubliquefrance.fr/maladies- } \\
\text { et-traumatismes/diabete }\end{array}$ & 2018 & Department \\
\hline \multicolumn{5}{|c|}{ Environmental factors } \\
\hline $\mathrm{PM}_{2.5}$ & $\begin{array}{l}\text { Particle matter } 2.5 \quad \text { (micro g } \\
/ \mathrm{m} 3 \text { ) }\end{array}$ & Global Ozone Monitoring Experiment (GOME) & $\begin{array}{l}2001- \\
2012\end{array}$ & Department \\
\hline Temperature & $\begin{array}{l}\text { Annual average of temperature } \\
\left({ }^{\circ} \mathrm{C}\right)\end{array}$ & $\begin{array}{l}\text { World climate } \\
\text { https://www.worldclim.org }\end{array}$ & $\begin{array}{l}2001- \\
2012\end{array}$ & Department \\
\hline
\end{tabular}

NB: The rate is standardized according to age, the reference population being the whole of France in the 2006 population census

Data on the availability of health services (number of resuscitation beds, number of intensive care beds, and medical density) at the departmental level were mainly extracted from the Direction of Research, Studies, Evaluation and Studies.

Data on socio-spatial factors, including demographic, socio-economic, and spatial characteristics, were obtained from the European Statistical Office and the National Federation of Regional Health Observatories (FNORS) (Supplementary Material Table 1). Data on the prevalence of diabetes were obtained from the FNORS.

\section{Measures}

\section{Outcome}

The COVID-19 mortality rate in hospitals at the departmental level was the primary health outcome of this study. It is defined as the number of COVID-19 deaths per 100,000 people.

\section{Covariates}

Air pollution exposure $\left(\mathrm{PM}_{2.5}\right)$ : The WHO limit is $10 \mu \mathrm{g} / \mathrm{m}^{3}$, but the EU guidelines indicate an annual mean air quality limit of $25 \mu \mathrm{g} / \mathrm{m}^{3}$ for $\mathrm{PM}_{2.5}$ since 2015, which is 2.5 times higher than the WHO limit [22]. 
The annual average $\mathrm{PM}_{2.5}$ concentration levels from 1999 to 2016 were computed to assess the potential association between long-term exposure to air pollution and the COVID-19 mortality rate. This period of measurement (i.e. annual average of exposure to $\mathrm{PM}_{2.5}$ ) considered in this study (>18 years) is longer than studies from Europe and USA [15-17, 23-25]. The annual average PM 2.5 concentration level was then defined in quartiles, thus making it possible to classify the departments according to the degree of their long-term exposure to air pollution (Supplementary Material Table 2).

Temperature: The annual average temperature (expressed in ${ }^{\circ} \mathrm{C}$ ) was obtained from the World Climate website, covering the $2001-2012$ period. Temperature is considered an environmental driver of several outbreaks, such as influenza. Shi et al. [18] found that temperature had a significant effect on the incidence of COVID-19. The authors showed that the transmission rate, which was correlated with the realtime temperature data, decreased as the temperature increased, leading to a reduction in the size and infection rate of the outbreak.

Availability of health services: The availability of health services such as resuscitation and intensive care units and physicians represents the health system's ability to manage patients with severe COVID-19. Here, it was considered in terms of the number of resuscitation and intensive care beds and medical density at the department level. These data were defined per 100,000 people.

Socio-demographic factors: In this study, the socio-demographic factors included demographic (proportion of people aged $\geq 60$ and the proportion of males), socioeconomic (proportion of adult people unemployed and poverty rate), and spatial or environmental characteristics (population density and the proportion of people living in large urban areas). However, this choice is constrained by the availability of information. These factors need to be carefully considered when estimating the association between air pollution and the incidence and mortality of COVID-19 [26].

Prevalence of diabetes: Diabetes was common among individuals who developed severe COVID-19 or died from it [27, 28]. It could represent a serious risk factor for COVID-19 in areas with a high prevalence rate. This health risk factor was calculated as the agestandardized prevalence rate per 100,000 individuals.

\section{Statistical analysis}

The characteristics of the dataset regarding the number of COVID-19 deaths, air pollution, health service availability, socio-spatial factors, and health status are described as mean, standard deviation, minimum, and maximum values. We explored the relationship between COVID-19 mortality with long-term $\mathrm{PM}_{2.5}$ exposure and temperature using correlation analysis. We analysed the geographic distribution of COVID-19 mortality rate at the department level depending on long-term exposure to $\mathrm{PM}_{2.5}$ grouped into quartiles. Next, we performed negative binomial regression analysis to examine whether long-term $\mathrm{PM}_{2.5}$ exposure and temperature were associated with the COVID-19 mortality rate, adjusted for several confounders (e.g. health service availability, health risk factors, and socio-demographic factors). We implemented nine separate models to estimate the effect of $\mathrm{PM}_{2.5}$ exposure and temperature on the COVID-19 mortality rate, adjusted for potential confounders mentioned above. These nine models corresponded to nine monthly dates considered during the period covered in this study. Furthermore, we performed negative binomial regression analysis to investigate the magnitude of disparities in COVID-19 mortality rate between departments with higher $\mathrm{PM}_{2.5}$ exposure levels and departments with lower $\mathrm{PM}_{2.5}$ exposure levels based on quartiles. This alternative modelling allowed us to show the robustness of the relationships between long-term $\mathrm{PM}_{2.5}$ exposure and the associated risk of COVID-19 mortality. The other analyses were performed as a robustness test, such as unadjusted regression analyses, to evaluate these relationships [29]. Negative binomial regression models allowed us to consider over-dispersion, which is often present in count data [30]. The multiplication coefficients of the negative binomial regression models corresponded to mortality rate ratios, which were interpreted as the percentage increase (or decrease) in the COVID-19 mortality rate associated with each quartile of long-term average $\mathrm{PM}_{2.5}$ exposure compared to the first quartile [31].

The results were assessed at a significant threshold of error of $5 \%$ or $95 \%$ confidence interval $(95 \% \mathrm{Cl})$. The parameter estimates from the negative binomial models were exponentiated to define the COVID-19 mortality rate ratio.

In addition, COVID-19 mortality maps were created to show the spatial disparities of the COVID-19 pandemic across metropolitan France using a free and open source as PhilCarto software (http://philcarto.free.fr) and Inkspace software (https://inkspace.org).

All analyses were performed using SAS software (release 9.4; SAS Institute Inc., Cary, NC, USA).

\section{Results}


The epidemiological situation observed in France until 31 December 2020 was worrying, and all the departments were not affected in the same way.

Table 2 presents the distribution characteristics of the parameters used in this study for each department in France.

As of 31 December 2020, the COVID-19 mortality rate in the department of metropolitan France was 65.4 per 100,000 inhabitants (standard deviation [SD] = 39.4) in hospitals. The annual average $\mathrm{PM}_{2.5}$ concentration level from 1999 to 2016 was $10.07 \%$ (SD = 2.40), and the annual average temperature from 2001 to 2012 was 10.67\% (SD =1.61). The average number of intensive care and resuscitation beds per 100,000 inhabitants was 14.09 (SD = 7.75), and the average medical density was $305.04(S D=89.33)$ per department.

The departments' socio-spatial characteristics showed that $29.58 \%$ of the population were aged $\geq 60$ years, $51.53 \%$ were women, $7.87 \%$ were unemployed, and $71.19 \%$ were living in large urban areas.

Table 2: Description of the study's characteristics, including Covid-19 deaths on 1 May and 31 December, 2020 , by mean (standard deviation), minimum and maximum

Mean (standard deviation) $\quad$ Min $\quad$ Max

COVID-19 deaths rate in hospitals (per 100,000):

- 1 May 2020

- 31 December 2020

\begin{tabular}{|c|c|c|c|}
\hline Annual Average long-term $\mathrm{PM}_{2.5}$ exposure from 1999 to $2016\left(\mu \mathrm{g} / \mathrm{m}^{3}\right)$ & $10.07(2.40)$ & 6.05 & 16.33 \\
\hline Annual average temperature over 12 years from 2001 to 2012 (in ${ }^{\circ} \mathrm{C}$ ) & $10.67(1.61)$ & 4.74 & 14.45 \\
\hline Number of intensive care and resuscitation beds (per 100,000 ) & $14.09(7.75)$ & 2.45 & 47.11 \\
\hline Medical density (per 100,000) & $305.04(89.33)$ & 167.00 & 858.00 \\
\hline \% People aged 60 or more & $29.58(4.83)$ & 16.68 & 39.32 \\
\hline$\%$ Males & $48.47(0.49)$ & 46.95 & 49.56 \\
\hline \% Unemployment & $7.9(1.6)$ & 4.8 & 13.3 \\
\hline Rate of poverty (per cent) & $14.64(3.11)$ & 9.20 & 28.60 \\
\hline \% Urban population (proportion of people living in the great urban areas) & $71.19(20.83)$ & 0.10 & 100.00 \\
\hline Population density (inhab/square) (by thousand) & $565.78(2425.06)$ & 14.76 & 20459.72 \\
\hline Population size (by thousand) & $676.02(520.06)$ & 76.29 & 2588.99 \\
\hline Standardized Prevalence of Diabetes at the department-level & $4.83(0.77)$ & 3.20 & 7.88 \\
\hline
\end{tabular}

The geographic distribution of COVID-19 mortality rates as a function of the annual average level of PM 2.5 , defined by quartiles, showed the existence of a gradient (see Fig 1). At the start of the period, the mortality rate due to COVID-19 in the departments classified in quartiles 1, 2, 3, and 4 were 6.6, 9.7, 24.6 and 38.3 deaths per 100,000 inhabitants, respectively. At the end of the period, during the second wave of the pandemic, the mortality rates were $41.3,52.8,80.4$, and 87.1 per 100,000 inhabitants, respectively.

The maps illustrate the dynamics of the spread of the COVID-19 pandemic across all departments compared to the annual average PM 2.5 concentration over the long term (Map 1).

Map 1: Geographical distribution of the COVID-19 mortality rate depending to quartile of PM 2.5 concentration level by department on 1 May 2020 (a), 1 July (b), and 31December 2020 (c)

Fig 1 and Map 1 show that the COVID-19 mortality rate increased with the level of particle matters concentration.

Table 3 shows the estimates of the effects of long-term exposure to $\mathrm{PM}_{2.5}$, and temperature on the COVID-19 mortality rates on different dates. The COVID-19 mortality rate was significantly associated with long-term exposure to air pollution until 1 November, and was no longer so in December 2020. Thus, a concentration of $1 \mu \mathrm{g} / \mathrm{m}^{3}$ of $\mathrm{PM}_{2.5}$, was associated with an increase in the COVID-19 mortality rate multiplied by a coefficient of 1.244, 1.264, 1.271, and 1.151, respectively, in May, July, September, and November 2020. The association between the COVID-19 mortality rate and temperature was not statistically significant until November 2020. The temperature was associated with a mortality rate multiplied by $0.905,0.870$, and 0.859 , respectively, on 1 November, 1 December, and 31 December 2020. 
Multiplier coefficients were less than 1 , which corresponded to a respective decrease in the mortality rate of $9.5 \%, 13.0 \%$, and $14.1 \%$ for a $1^{\circ} \mathrm{C}$ increase in the annual average temperature between the departments.

Table 3: Estimates effects of long-term $\mathrm{PM}_{2.5}$ exposure and Temperature on COVID-19 mortality rate using the negative binomial regression model (From 01 May to 31 December, 2020) 


\begin{tabular}{|c|c|c|c|c|c|c|c|c|c|}
\hline $\begin{array}{l}\text { Independent } \\
\text { variables }\end{array}$ & 01 May & 01 June & 01 July & $\begin{array}{c}01 \\
\text { August }\end{array}$ & $\begin{array}{c}01 \\
\text { September }\end{array}$ & $\begin{array}{c}01 \\
\text { October }\end{array}$ & $\begin{array}{c}01 \\
\text { November } \\
\end{array}$ & $\begin{array}{c}01 \\
\text { December } \\
\end{array}$ & $\begin{array}{c}31 \\
\text { December } \\
\end{array}$ \\
\hline $\begin{array}{l}\text { Annual } \\
\text { average Long- } \\
\text { term } \mathrm{PM}_{2.5} \\
\text { exposure } \\
\left(\mu \mathrm{g} / \mathrm{m}^{3}\right)\end{array}$ & $\begin{array}{l}1.244^{*} \\
(1.083- \\
1.428)\end{array}$ & $\begin{array}{c}1.258^{* *} \\
(1.104- \\
1.434)\end{array}$ & $\begin{array}{c}1.264^{* *} \\
(1.111- \\
1.439)\end{array}$ & $\begin{array}{c}1.267 * * \\
(1.114- \\
1.442)\end{array}$ & $\begin{array}{c}1.271 * * \\
(1.118- \\
1.444)\end{array}$ & $\begin{array}{l}1.258^{* *} \\
(1.111- \\
1.423)\end{array}$ & $\begin{array}{c}1.151 * * \\
(1.036- \\
1.279)\end{array}$ & $\begin{array}{c}1.078 \\
(0.985- \\
1.180)\end{array}$ & $\begin{array}{l}1.090 \\
(0.999- \\
1.190)\end{array}$ \\
\hline $\begin{array}{l}\text { Annual } \\
\text { average } \\
\text { Temperature } \\
\text { over } 12 \text { years }\end{array}$ & $\begin{array}{c}0.916 \\
(0.829- \\
1.012)\end{array}$ & $\begin{array}{c}0.913 \\
(0.832- \\
1.002)\end{array}$ & $\begin{array}{c}0.913 \\
(0.832- \\
1.001)\end{array}$ & $\begin{array}{c}0.914 \\
(0.834- \\
1.002)\end{array}$ & $\begin{array}{c}0.917 \\
(0.837- \\
1.004)\end{array}$ & $\begin{array}{c}0.921 \\
(0.844- \\
1.005)\end{array}$ & $\begin{array}{c}0.905 * * \\
(0.840- \\
0.974)\end{array}$ & $\begin{array}{c}0.870^{* *} \\
(0.816- \\
0.927)\end{array}$ & $\begin{array}{l}0.859 * * \\
(0.807- \\
0.914)\end{array}$ \\
\hline $\begin{array}{l}\text { Number of } \\
\text { intensive care } \\
\text { and } \\
\text { resuscitation } \\
\text { beds (per } \\
100,000 \text { ) }\end{array}$ & $\begin{array}{l}1.025^{*} \\
(1.002- \\
1.049)\end{array}$ & $\begin{array}{c}1.020 \\
(0.999- \\
1.042)\end{array}$ & $\begin{array}{c}1.019 \\
(0.998- \\
1.041)\end{array}$ & $\begin{array}{l}1.018 \\
(0.997- \\
1.040)\end{array}$ & $\begin{array}{c}1.018 \\
(0.997- \\
1.039)\end{array}$ & $\begin{array}{l}1.015 \\
(0.995- \\
1.036)\end{array}$ & $\begin{array}{l}1.011 \\
(0.994- \\
1.029)\end{array}$ & $\begin{array}{c}1.003 \\
(0.988- \\
1.018)\end{array}$ & $\begin{array}{c}1.006 \\
(0.991- \\
1.020)\end{array}$ \\
\hline $\begin{array}{l}\text { Medical } \\
\text { density (per } \\
100,000)\end{array}$ & $\begin{array}{c}0.998 \\
(0.995- \\
1.000) \\
\end{array}$ & $\begin{array}{c}0.999 \\
(0.996- \\
1.001) \\
\end{array}$ & $\begin{array}{c}0.999 \\
(0.997- \\
1.001) \\
\end{array}$ & $\begin{array}{c}0.999 \\
(0.997- \\
1.001) \\
\end{array}$ & $\begin{array}{c}0.999 \\
(0.997- \\
1.001) \\
\end{array}$ & $\begin{array}{c}1.000 \\
(0.997- \\
1.002) \\
\end{array}$ & $\begin{array}{c}1.000 \\
(0.998- \\
1.002) \\
\end{array}$ & $\begin{array}{c}1.001 \\
(0.999- \\
1.002) \\
\end{array}$ & $\begin{array}{c}1.001 \\
(0.999- \\
1.002) \\
\end{array}$ \\
\hline $\begin{array}{l}\% \text { People aged } \\
60 \text { or more }\end{array}$ & $\begin{array}{c}1.088^{* *} \\
(1.021- \\
1.158) \\
\end{array}$ & $\begin{array}{c}1.096 * * \\
(1.032- \\
1.163) \\
\end{array}$ & $\begin{array}{c}1.097 * * \\
(1.034- \\
1.164) \\
\end{array}$ & $\begin{array}{c}1.100 * * \\
(1.037- \\
1.167) \\
\end{array}$ & $\begin{array}{c}1.097 * * \\
(1.035- \\
1.163) \\
\end{array}$ & $\begin{array}{c}1.096 * * \\
(1.036- \\
1.159) \\
\end{array}$ & $\begin{array}{c}1.046 \\
(0.999- \\
1.095) \\
\end{array}$ & $\begin{array}{c}1.037 \\
(0.997- \\
1.080) \\
\end{array}$ & $\begin{array}{c}1.039 \\
(0.999- \\
1.080) \\
\end{array}$ \\
\hline$\%$ Males & $\begin{array}{c}1.467 \\
(0.995- \\
2.164) \\
\end{array}$ & $\begin{array}{l}1.521 * \\
(1.050- \\
2.203) \\
\end{array}$ & $\begin{array}{l}1.557^{*} \\
(1.079- \\
2.248) \\
\end{array}$ & $\begin{array}{l}1.589 * \\
(1.102- \\
2.291) \\
\end{array}$ & $\begin{array}{l}1.585^{*} \\
(1.103- \\
2.277) \\
\end{array}$ & $\begin{array}{l}1.571 * \\
(1.106- \\
2.233) \\
\end{array}$ & $\begin{array}{l}1.482 * \\
(1.098- \\
1.999) \\
\end{array}$ & $\begin{array}{l}1.331 * \\
(1.025- \\
1.730) \\
\end{array}$ & $\begin{array}{l}1.310^{*} \\
(1.015- \\
1.690) \\
\end{array}$ \\
\hline $\begin{array}{l}\% \\
\text { Unemployment }\end{array}$ & $\begin{array}{c}1.011 \\
(0.872- \\
1.173) \\
\end{array}$ & $\begin{array}{c}0.996 \\
(0.866- \\
1.145) \\
\end{array}$ & $\begin{array}{c}0.997 \\
(0.868- \\
1.145) \\
\end{array}$ & $\begin{array}{c}0.997 \\
(0.868- \\
1.145) \\
\end{array}$ & $\begin{array}{c}1.001 \\
(0.873- \\
1.148) \\
\end{array}$ & $\begin{array}{c}0.974 \\
(0.854- \\
1.110) \\
\end{array}$ & $\begin{array}{c}0.987 \\
(0.883- \\
1.105) \\
\end{array}$ & $\begin{array}{c}0.989 \\
(0.897- \\
1.091) \\
\end{array}$ & $\begin{array}{c}0.990 \\
(0.899- \\
1.090) \\
\end{array}$ \\
\hline $\begin{array}{l}\text { Rate of Poverty } \\
\text { (per cent) }\end{array}$ & $\begin{array}{c}0.999 \\
(0.904- \\
1.104) \\
\end{array}$ & $\begin{array}{c}1.006 \\
(0.916- \\
1.105) \\
\end{array}$ & $\begin{array}{c}1.009 \\
(0.920- \\
1.107) \\
\end{array}$ & $\begin{array}{c}1.010 \\
(0.921- \\
1.108) \\
\end{array}$ & $\begin{array}{c}1.009 \\
(0.921- \\
1.106) \\
\end{array}$ & $\begin{array}{c}1.026 \\
(0.940- \\
1.120) \\
\end{array}$ & $\begin{array}{c}1.023 \\
(0.948- \\
1.103) \\
\end{array}$ & $\begin{array}{c}1.018 \\
(0.953- \\
1.087) \\
\end{array}$ & $\begin{array}{c}1.017 \\
(0.954- \\
1.084) \\
\end{array}$ \\
\hline $\begin{array}{l}\text { \% Urban } \\
\text { population } \\
\text { (proportion of } \\
\text { people living } \\
\text { in the great } \\
\text { urban areas) } \\
\end{array}$ & $\begin{array}{c}1.021 * * \\
(1.006- \\
1.037)\end{array}$ & $\begin{array}{c}1.021 * * \\
(1.007- \\
1.036)\end{array}$ & $\begin{array}{c}1.021 * * \\
(1.007- \\
1.036)\end{array}$ & $\begin{array}{c}1.022 * * \\
(1.008- \\
1.037)\end{array}$ & $\begin{array}{l}1.021 * * \\
(1.007- \\
1.036)\end{array}$ & $\begin{array}{c}1.023^{* *} \\
(1.009- \\
1.036)\end{array}$ & $\begin{array}{l}1.013^{*} \\
(1.002- \\
1.024)\end{array}$ & $\begin{array}{l}1.011 * \\
(1.002- \\
1.020)\end{array}$ & $\begin{array}{l}1.008 \\
(0.999- \\
1.017)\end{array}$ \\
\hline $\begin{array}{l}\text { Population } \\
\text { density } \\
\text { (inhab/square) }\end{array}$ & $\begin{array}{l}1.000 \\
(1.000- \\
1.000) \\
\end{array}$ & $\begin{array}{l}1.000 \\
(1.000- \\
1.000) \\
\end{array}$ & $\begin{array}{l}1.000 \\
(1.000- \\
1.000) \\
\end{array}$ & $\begin{array}{c}1.000 \\
(1.000- \\
1.000) \\
\end{array}$ & $\begin{array}{l}1.000 \\
(1.000- \\
1.000) \\
\end{array}$ & $\begin{array}{l}1.000 \\
(1.000- \\
1.000) \\
\end{array}$ & $\begin{array}{l}1.000 \\
(1.000- \\
1.000) \\
\end{array}$ & $\begin{array}{l}1.000 \\
(1.000- \\
1.000) \\
\end{array}$ & $\begin{array}{l}1.000 \\
(1.000- \\
1.000) \\
\end{array}$ \\
\hline $\begin{array}{l}\text { Standardized } \\
\text { Prevalence of } \\
\text { Diabetes (\%) }\end{array}$ & $\begin{array}{c}1.160 \\
(0.772- \\
1.742) \\
\end{array}$ & $\begin{array}{c}1.136 \\
(0.775- \\
1.666) \\
\end{array}$ & $\begin{array}{c}1.138 \\
(0.779- \\
1.661) \\
\end{array}$ & $\begin{array}{l}1.134 \\
(0.777- \\
1.653) \\
\end{array}$ & $\begin{array}{c}1.125 \\
(0.775- \\
1.633) \\
\end{array}$ & $\begin{array}{l}1.126 \\
(0.785- \\
1.614) \\
\end{array}$ & $\begin{array}{c}1.187 \\
(0.871- \\
1.619) \\
\end{array}$ & $\begin{array}{c}1.216 \\
(0.930- \\
1.589) \\
\end{array}$ & $\begin{array}{c}1.188 \\
(0.916- \\
1.542) \\
\end{array}$ \\
\hline \multicolumn{10}{|c|}{ Criteria for assessing goodness of fit: } \\
\hline Deviance & 1.1594 & 1.1835 & 1.1859 & 1.1882 & 1.1841 & 1.1897 & 1.1574 & 1.171 & 1.1661 \\
\hline $\begin{array}{l}\text { Scaled } \\
\text { Deviance }\end{array}$ & 1.1594 & 1.1835 & 1.1859 & 1.1882 & 1.1841 & 1.1897 & 1.1574 & 1.171 & 1.1661 \\
\hline $\begin{array}{l}\text { Pearson Chi- } \\
\text { Square }\end{array}$ & 1.3409 & 1.2943 & 1.2899 & 1.2881 & 1.2894 & 1.2474 & 1.2029 & 1.1794 & 1.2021 \\
\hline $\begin{array}{l}\text { Scaled } \\
\text { Pearson X2 }\end{array}$ & 1.3409 & 1.2943 & 1.2899 & 1.2881 & 1.2894 & 1.2474 & 1.2029 & 1.1794 & 1.2021 \\
\hline $\begin{array}{l}\text { BIC (smaller is } \\
\text { better) }\end{array}$ & 731.9157 & 765.3375 & 771.5867 & 774.6766 & 776.8832 & 784.2321 & 805.7196 & 879.2935 & 924.3351 \\
\hline
\end{tabular}

(*)Significance at 5\%, (**) Significance at 1\%

Table 4 shows results of the $\mathrm{PM}_{2.5}$ concentration level defined in the quartile. The risk of death from COVID-19 was higher in departments with a $\mathrm{PM}_{2.5}$ concentration level equal to or greater than the third quartile, compared to those located in the first quartile. For example, 
departments with levels of $\mathrm{PM}_{2.5}$ exposure in the third quartile had 2.408, 2.295, 1.624, and 1.469 times greater risk of death from COVID19 than departments placed in the first quartile, respectively on 1 May, 1 August, 1 November, and 31 December 2020. Note the absence of a statistically significant difference in the mortality rate between the departments with $\mathrm{PM}_{2.5}$ concentration level belonging to the first and the second quartile.

For the association between the COVID-19 mortality rates and temperature, the results were stable and significant from 1 November.

Table 4: Estimates effects of long-term $\mathrm{PM}_{2.5}$ exposure in quartile and Temperature on COVID-19 mortality rate using the negative binomial regression model (From 01May to 31 December, 2020) 


\begin{tabular}{|c|c|c|c|c|c|c|c|c|c|}
\hline $\begin{array}{l}\text { Independent } \\
\text { variables }\end{array}$ & 01 May & 01 June & 01 July & $\begin{array}{c}01 \\
\text { August } \\
\end{array}$ & $\begin{array}{c}01 \\
\text { September } \\
\end{array}$ & $\begin{array}{c}01 \\
\text { October } \\
\end{array}$ & $\begin{array}{c}01 \\
\text { November } \\
\end{array}$ & $\begin{array}{c}01 \\
\text { December } \\
\end{array}$ & $\begin{array}{c}31 \\
\text { December } \\
\end{array}$ \\
\hline \multicolumn{10}{|l|}{$\begin{array}{l}\text { Annual average } \\
\text { Long-term } \mathrm{PM}_{2.5} \\
\text { exposure }\left(\mu \mathrm{g} / \mathrm{m}^{3}\right) \text { in } \\
\text { quartile (ref. =Q1) }\end{array}$} \\
\hline Q2 & $\begin{array}{l}1.134 \\
(0.758- \\
1.699) \\
\end{array}$ & $\begin{array}{l}1.144 \\
(0.779- \\
1.680) \\
\end{array}$ & $\begin{array}{l}1.134 \\
(0.775- \\
1.660) \\
\end{array}$ & $\begin{array}{l}1.127 \\
(0.771- \\
1.649) \\
\end{array}$ & $\begin{array}{c}1.126 \\
(0.773- \\
1.640) \\
\end{array}$ & $\begin{array}{l}1.144 \\
(0.796- \\
1.643) \\
\end{array}$ & $\begin{array}{l}1.105 \\
(0.807- \\
1.513) \\
\end{array}$ & $\begin{array}{l}1.142 \\
(0.876- \\
1.490) \\
\end{array}$ & $\begin{array}{l}1.190 \\
(0.922- \\
1.535) \\
\end{array}$ \\
\hline Q3 & $\begin{array}{c}2.408 * * \\
(1.508- \\
3.845) \\
\end{array}$ & $\begin{array}{l}2.302 * * \\
(1.474- \\
3.597) \\
\end{array}$ & $\begin{array}{c}2.307^{* *} \\
(1.484- \\
3.586) \\
\end{array}$ & $\begin{array}{c}2.295^{* *} \\
(1.476- \\
3.568) \\
\end{array}$ & $\begin{array}{l}2.317^{* *} \\
(1.498- \\
3.582) \\
\end{array}$ & $\begin{array}{l}2.234^{* *} \\
(1.467- \\
3.404) \\
\end{array}$ & $\begin{array}{l}1.624^{* *} \\
(1.129- \\
2.336) \\
\end{array}$ & $\begin{array}{l}1.366^{*} \\
(1.007- \\
1.853) \\
\end{array}$ & $\begin{array}{l}1.469 * * \\
(1.099- \\
1.963) \\
\end{array}$ \\
\hline Q4 & $\begin{array}{c}2.797 * * \\
(1.439- \\
5.436) \\
\end{array}$ & $\begin{array}{l}2.678^{* *} \\
(1.414- \\
5.070) \\
\end{array}$ & $\begin{array}{c}2.697 * * \\
(1.433- \\
5.074) \\
\end{array}$ & $\begin{array}{l}2.682^{* *} \\
(1.424- \\
5.052) \\
\end{array}$ & $\begin{array}{c}2.680^{* *} \\
(1.433- \\
5.012) \\
\end{array}$ & $\begin{array}{c}2.596 * * \\
(1.416- \\
4.760) \\
\end{array}$ & $\begin{array}{l}1.712^{*} \\
(1.013- \\
2.893) \\
\end{array}$ & $\begin{array}{l}1.141 \\
(0.734- \\
1.776) \\
\end{array}$ & $\begin{array}{l}1.140 \\
(0.749- \\
1.736) \\
\end{array}$ \\
\hline $\begin{array}{l}\text { Annual average } \\
\text { Temperature over } 12 \\
\text { years }\end{array}$ & $\begin{array}{c}0.919 \\
(0.835- \\
1.012) \\
\end{array}$ & $\begin{array}{c}0.914 \\
(0.835- \\
1.001) \\
\end{array}$ & $\begin{array}{l}0.914^{*} \\
(0.836- \\
1.000) \\
\end{array}$ & $\begin{array}{c}0.915 \\
(0.837- \\
1.001) \\
\end{array}$ & $\begin{array}{c}0.918 \\
(0.841- \\
1.003) \\
\end{array}$ & $\begin{array}{c}0.921 \\
(0.846- \\
1.004) \\
\end{array}$ & $\begin{array}{l}0.903 * * \\
(0.839- \\
0.972) \\
\end{array}$ & $\begin{array}{l}0.867 * * \\
(0.814- \\
0.923) \\
\end{array}$ & $\begin{array}{l}0.855^{* *} \\
(0.805- \\
0.908) \\
\end{array}$ \\
\hline $\begin{array}{l}\text { Number of intensive } \\
\text { care and } \\
\text { resuscitation beds } \\
\text { (per 100,000) }\end{array}$ & $\begin{array}{l}1.015 \\
(0.991- \\
1.039)\end{array}$ & $\begin{array}{l}1.010 \\
(0.988- \\
1.033)\end{array}$ & $\begin{array}{l}1.009 \\
(0.987- \\
1.031)\end{array}$ & $\begin{array}{l}1.008 \\
(0.986- \\
1.031)\end{array}$ & $\begin{array}{l}1.008 \\
(0.986- \\
1.030)\end{array}$ & $\begin{array}{l}1.006 \\
(0.985- \\
1.027)\end{array}$ & $\begin{array}{l}1.006 \\
(0.987- \\
1.024)\end{array}$ & $\begin{array}{l}1.000 \\
(0.984- \\
1.015)\end{array}$ & $\begin{array}{l}1.001 \\
(0.986- \\
1.017)\end{array}$ \\
\hline $\begin{array}{l}\text { Medical density (per } \\
100,000 \text { ) }\end{array}$ & $\begin{array}{c}0.998 \\
(0.996- \\
1.001) \\
\end{array}$ & $\begin{array}{c}0.999 \\
(0.997- \\
1.001) \\
\end{array}$ & $\begin{array}{c}0.999 \\
(0.997- \\
1.001) \\
\end{array}$ & $\begin{array}{c}0.999 \\
(0.997- \\
1.002) \\
\end{array}$ & $\begin{array}{c}0.999 \\
(0.997- \\
1.002) \\
\end{array}$ & $\begin{array}{c}1.000 \\
(0.998- \\
1.002) \\
\end{array}$ & $\begin{array}{c}1.000 \\
(0.998- \\
1.002) \\
\end{array}$ & $\begin{array}{c}1.001 \\
(0.999- \\
1.002) \\
\end{array}$ & $\begin{array}{c}1.001 \\
(0.999- \\
1.002) \\
\end{array}$ \\
\hline $\begin{array}{l}\% \text { People aged } 60 \text { or } \\
\text { more }\end{array}$ & $\begin{array}{l}1.073^{*} \\
(1.009- \\
1.140) \\
\end{array}$ & $\begin{array}{l}1.078^{*} \\
(1.017- \\
1.144) \\
\end{array}$ & $\begin{array}{c}1.080 * * \\
(1.019- \\
1.145) \\
\end{array}$ & $\begin{array}{c}1.083^{* *} \\
(1.021- \\
1.148) \\
\end{array}$ & $\begin{array}{l}1.079^{*} \\
(1.018- \\
1.143) \\
\end{array}$ & $\begin{array}{c}1.079 * * \\
(1.020- \\
1.141) \\
\end{array}$ & $\begin{array}{c}1.032 \\
(0.986- \\
1.081) \\
\end{array}$ & $\begin{array}{c}1.017 \\
(0.977- \\
1.059) \\
\end{array}$ & $\begin{array}{c}1.013 \\
(0.975- \\
1.053) \\
\end{array}$ \\
\hline$\%$ Males & $\begin{array}{c}1.436 \\
(0.986- \\
2.092) \\
\end{array}$ & $\begin{array}{l}1.494^{*} \\
(1.040- \\
2.147) \\
\end{array}$ & $\begin{array}{l}1.530 * \\
(1.068- \\
2.191) \\
\end{array}$ & $\begin{array}{l}1.561 * \\
(1.091- \\
2.235) \\
\end{array}$ & $\begin{array}{l}1.559 * \\
(1.093- \\
2.223) \\
\end{array}$ & $\begin{array}{l}1.543^{*} \\
(1.092- \\
2.180) \\
\end{array}$ & $\begin{array}{l}\text { 1.456* } \\
\text { (1.080- } \\
1.963) \\
\end{array}$ & $\begin{array}{l}1.308^{*} \\
(1.010- \\
1.694) \\
\end{array}$ & $\begin{array}{c}1.278 \\
(0.997- \\
1.637) \\
\end{array}$ \\
\hline \% Unemployment & $\begin{array}{c}1.027 \\
(0.889- \\
1.186) \\
\end{array}$ & $\begin{array}{c}1.014 \\
(0.884- \\
1.163) \\
\end{array}$ & $\begin{array}{c}1.015 \\
(0.886- \\
1.163) \\
\end{array}$ & $\begin{array}{c}1.015 \\
(0.886- \\
1.164) \\
\end{array}$ & $\begin{array}{c}1.020 \\
(0.891- \\
1.167) \\
\end{array}$ & $\begin{array}{c}0.989 \\
(0.870- \\
1.126) \\
\end{array}$ & $\begin{array}{c}0.997 \\
(0.891- \\
1.115) \\
\end{array}$ & $\begin{array}{c}1.008 \\
(0.914- \\
1.111) \\
\end{array}$ & $\begin{array}{c}1.014 \\
(0.924- \\
1.114) \\
\end{array}$ \\
\hline $\begin{array}{l}\text { Rate of Poverty (per } \\
\text { cent) }\end{array}$ & $\begin{array}{c}1.000 \\
(0.910- \\
1.100) \\
\end{array}$ & $\begin{array}{c}1.000 \\
(0.914- \\
1.094) \\
\end{array}$ & $\begin{array}{c}1.002 \\
(0.917- \\
1.096) \\
\end{array}$ & $\begin{array}{c}1.002 \\
(0.916- \\
1.095) \\
\end{array}$ & $\begin{array}{c}1.000 \\
(0.916- \\
1.093) \\
\end{array}$ & $\begin{array}{c}1.018 \\
(0.935- \\
1.109) \\
\end{array}$ & $\begin{array}{c}1.017 \\
(0.944- \\
1.095) \\
\end{array}$ & $\begin{array}{c}1.006 \\
(0.944- \\
1.072) \\
\end{array}$ & $\begin{array}{c}1.003 \\
(0.944- \\
1.065) \\
\end{array}$ \\
\hline $\begin{array}{l}\text { \% Urban population } \\
\text { (proportion of } \\
\text { people living in the } \\
\text { great urban areas) }\end{array}$ & $\begin{array}{c}1.025^{* *} \\
(1.009- \\
1.040)\end{array}$ & $\begin{array}{l}1.024^{* *} \\
(1.009- \\
1.039)\end{array}$ & $\begin{array}{l}1.024 * * \\
(1.010- \\
1.039)\end{array}$ & $\begin{array}{l}1.025 * * \\
(1.010- \\
1.040)\end{array}$ & $\begin{array}{l}1.024^{* *} \\
(1.010- \\
1.039)\end{array}$ & $\begin{array}{c}1.025^{* *} \\
(1.011- \\
1.039)\end{array}$ & $\begin{array}{l}1.014 * \\
(1.003- \\
1.026)\end{array}$ & $\begin{array}{c}1.009 \\
(0.999- \\
1.019)\end{array}$ & $\begin{array}{c}1.006 \\
(0.996- \\
1.015)\end{array}$ \\
\hline $\begin{array}{l}\text { Population density } \\
\text { (inhab/square) }\end{array}$ & $\begin{array}{l}1.000^{* *} \\
(1.000- \\
1.000) \\
\end{array}$ & $\begin{array}{c}1.000 * * \\
(1.000- \\
1.000) \\
\end{array}$ & $\begin{array}{c}1.000 * * \\
(1.000- \\
1.000) \\
\end{array}$ & $\begin{array}{c}1.000 * * \\
(1.000- \\
1.000) \\
\end{array}$ & $\begin{array}{c}1.000 * * \\
(1.000- \\
1.000) \\
\end{array}$ & $\begin{array}{l}1.000 * \\
(1.000- \\
1.000) \\
\end{array}$ & $\begin{array}{l}1.000 * \\
(1.000- \\
1.000) \\
\end{array}$ & $\begin{array}{l}1.000 * \\
(1.000- \\
1.000) \\
\end{array}$ & $\begin{array}{l}1.000 * \\
(1.000- \\
1.000) \\
\end{array}$ \\
\hline $\begin{array}{l}\text { Standardized } \\
\text { Prevalence of } \\
\text { Diabetes (\%) }\end{array}$ & $\begin{array}{c}1.191 \\
(0.831- \\
1.708) \\
\end{array}$ & $\begin{array}{c}1.235 \\
(0.879- \\
1.735) \\
\end{array}$ & $\begin{array}{c}1.247 \\
(0.890- \\
1.745) \\
\end{array}$ & $\begin{array}{l}1.253 \\
(0.895- \\
1.753) \\
\end{array}$ & $\begin{array}{c}1.250 \\
(0.897- \\
1.741) \\
\end{array}$ & $\begin{array}{c}1.244 \\
(0.901- \\
1.717) \\
\end{array}$ & $\begin{array}{c}1.288 \\
(0.973- \\
1.705) \\
\end{array}$ & $\begin{array}{l}1.353^{*} \\
(1.066- \\
1.716) \\
\end{array}$ & $\begin{array}{l}1.352^{* *} \\
(1.077- \\
1.698) \\
\end{array}$ \\
\hline \multicolumn{10}{|c|}{ Criteria for assessing goodness of fit: } \\
\hline Deviance & 1.1782 & 1.2091 & 1.2114 & 1.2134 & 1.2086 & 1.2147 & 1.1827 & 1.1982 & 1.1927 \\
\hline Scaled Deviance & 1.1782 & 1.2091 & 1.2114 & 1.2134 & 1.2086 & 1.2147 & 1.1827 & 1.1982 & 1.1927 \\
\hline Pearson Chi-Square & 1.2419 & 1.2275 & 1.2168 & 1.2148 & 1.2164 & 1.1875 & 1.1898 & 1.1747 & 1.1834 \\
\hline Scaled Pearson $X 2$ & 1.2419 & 1.2275 & 1.2168 & 1.2148 & 1.2164 & 1.1875 & 1.1898 & 1.1747 & 1.1834 \\
\hline $\begin{array}{l}\text { BIC (smaller is } \\
\text { better) }\end{array}$ & 733.6727 & 769.6559 & 775.9792 & 779.4617 & 781.455 & 789.3626 & 813.6141 & 885.5104 & 927.3749 \\
\hline
\end{tabular}

${ }^{*}$ Significance at 5\%, (**) Significance at 1\% 
In addition, the results showed that the adjustment variables related to the availability of health resources and socio-economic factors did not have an overall influence on the COVID-19 mortality rate between departments. In contrast, demographic variables and urbanisation rates were statistically associated with the COVID-19 mortality rate for several months.

\section{Discussion}

This study provides significant findings on the associations between COVID-19 mortality rates, long-term exposure to $\mathrm{PM}_{2.5}$, and temperature in the context of socio-spatial disparity across the ninety-six departments of France.

\section{Spatial distribution of COVID-19 mortality rate and environmental factors}

The geographic distribution of annual average $\mathrm{PM}_{2.5}$ concentration levels across France was uneven and reflected the distribution of emission sources and urbanisation rate of each department.

Our results showed an increasing trend in the distribution of COVID-19 mortality rates across the departments grouped into quartiles of the annual average level of $\mathrm{PM}_{2.5}$. Indeed, despite the gradual spread of the COVID-19 pandemic across the whole country, the mortality rate followed a gradient determined by the level of $\mathrm{PM}_{2.5}$, as shown in Fig. 1. They were all higher in the departments where the annual average of long-term $\mathrm{PM}_{2.5}$ concentrations was also high. Our results highlighted the existence of a strong correlation between the mortality rate due to COVID-19 and long-term exposure to $\mathrm{PM}_{2.5}$.

These results overlapped well with the COVID-19 incidence data published regularly by the Ministry of Health. Considered separately, some departments could present an epidemiological picture different from the group to which it was assigned based on its level of $\mathrm{PM}_{2.5}$ concentration. The spatial distribution of COVID-19 cases and deaths is not random and may be related to environmental factors [32].

These geographic disparities in COVID-19 mortality rates, depending on the level of long-term $\mathrm{PM}_{2.5}$, could be related to other parameters such as the pandemic's spatial dynamics, the intensity of social interactions, and aggregation of infected individuals [33]. These disparities could also be associated with the differential vulnerability between each sub-national level, such as the existence of medical conditions [2,27] and socio-economic conditions. Environmental factors and various vulnerabilities could represent a favourable context for the spread of the COVID-19 pandemic and the increase in COVID-19 deaths [17]. The maps made it possible to observe the evolution (or trend) of the COVID-19 mortality rate across departments, given their levels of long-term exposure to $\mathrm{PM}_{2.5}$, expressed in quartiles.

Other studies have shown significant correlations between long-term exposure to $\mathrm{PM}_{2.5}$, and the incidence or mortality due to the COVID19 pandemic [17, 34]. Pansini and Fornacca [34] concluded that higher mortality was also correlated with poor air quality, namely, high $\mathrm{PM}_{2.5}$. In this new study, significant positive correlations of the COVID-19 mortality rate were found with long-term exposure to $\mathrm{PM}_{2.5}$, urbanisation rate, population density, and standardised prevalence rate of diabetes. In contrast, negative correlations were found with long-term annual average temperature.

\section{Association between COVID-19 mortality rate and long-term $\mathrm{PM}_{2.5}$ exposure}

The existence of an association between the risk of death and hospitalisation for COVID-19 and long-term exposure to $\mathrm{PM}_{2.5}$ indicates the role of air pollution in the development of an increased vulnerability of specific populations to COVID -19, especially the elderly, men, and people living in large urban areas. We observed a positive gradient in the COVID-19 mortality rate linked to the annual average level of $\mathrm{PM}_{2.5}$, when defined by quartile.

Our study showed the stability of the impact of long-term exposure to $\mathrm{PM}_{2.5}$ on the COVID-19 mortality rate, varying from 1.244 (or $24.4 \%$ ) to 1.258 (or $25.8 \%$ ) on May 1 to October 1, respectively, before falling to 1.151 (or $15.1 \%$ ) on 1 November then ceased to be statistically significant on December 1 and 31 2020. The beginning of the decrease in the intensity of the association between long-term exposure to

$\mathrm{PM}_{2.5}$, and the COVID-19 mortality rate seemed to coincide with the pandemic's resurgence in September (see Supplementary Material Fig 1), also called the second wave. This second wave amplified the spread of SARS-CoV-2 through departments that were slightly affected and had lower $\mathrm{PM}_{2.5}$. This would have gradually reduced the specific effects of geographic disparities in pollution levels between departments.

On the other hand, considering the distribution of long-term exposure to $\mathrm{PM}_{2.5}$, our results revealed a positive gradient in the impact of air pollution on the COVID-19 mortality rate from the third quartile. This gradient was observed until 1 November 2020. From December 1 , 
only the departments belonging to the third quartile of the $\mathrm{PM}_{2.5}$ concentration level had higher COVID-19 mortality rates than those in the first quartile. This result is important as it suggests that the effect of long-term exposure to $\mathrm{PM}_{2.5}$ on the COVID-19 mortality rate was not significant in France until the end of 2020. In addition, this effect remained associated with the geographic disparities in mortality rates observed when less comprehensive analyses could be performed. It should also be noted that there was no statistically significant difference in the COVID-19 mortality rates between the departments located in the first and the second quartiles. Since the start of the SARS-CoV-2 pandemic, several studies have advanced various hypotheses that may help to understand the mechanisms by which exposure to $\mathrm{PM}_{2.5}$, and air pollution in general, would influence the spread of the coronavirus and would induce the severity of infected cases and deaths [35-38]. For example, Wang et al. [39] considered that $\mathrm{PM}_{2.5}$ could facilitate SARS-CoV-2 infection through the overregulation of ACE2. As the impact of air pollution on health is well documented, the association between long-term air pollution and the severity of infected cases and mortality is thought to be mediated by the various morbidity conditions caused by chronic exposure to poor air quality.

This association could be explained by the fact that short- and long-term exposure to air pollution in the population was linked to asthma attacks, exacerbation of COPD, acute respiratory inflammation, and cardiorespiratory disease linked to death. Prolonged exposure to air pollution leads to a chronic inflammatory stimulus, even in young and healthy subjects, and could induce persistent modifications of the immune system, for which short-term changes in air quality may not be sufficient to break the aforementioned vicious circle [24]. Of course, the latter authors have observed the persistence of a high fatality rate, despite the dramatic reduction in air pollution levels in Lombardy since the start of the outbreak. Our results showed certain stability of the effects of long-term exposure to PM 2.5 , on the COVID19 mortality rates. These results are consistent with those obtained by other studies in the USA, Italy, China, Great Britain, the Netherlands, and Spain.

In the USA, Wu et al. [13] found that an increase of only $1 \mu \mathrm{g} / \mathrm{m}^{3}$ in long-term average $\mathrm{PM}_{2.5}$, is associated with a statistically significant increase of $15 \%$ in the COVID-19 mortality rate with data collected on 5 April 2020. Using data from 18 June 2020 the same authors found that the association led to an increase of $11 \%$ in the COVID-19 mortality rate [8].

In the Netherlands, Cole et al. [40] examined COVID-19 data between February and June 2020 and found a statistically significant positive relationship between long-term $\mathrm{PM}_{2.5}$ exposure and COVID-19 deaths. Their findings indicated that an increase in $\mathrm{PM}_{2.5}$ concentrations of $1 \mu \mathrm{g} / \mathrm{m}^{3}$ was associated with an increase in COVID-19 deaths of between $13.0 \%$ and $21.4 \%$.

Coker et al. [15] found a $1 \mu \mathrm{g} / \mathrm{m}^{3}$ increase in $\mathrm{PM}_{2.5}$, leading to a $9 \%$ increase in COVID-19 related excess mortality at the municipality level.

In a study concerning the regional and global contributions of air pollution to the risk of death from COVID-19, Pozzer et al. [37] found that the COVID-19 mortality fraction attributed to air pollution was $11 \%$ for fossil fuel-related emissions in France, $17 \%$ in Germany, $12 \%$ in Italy, and $9 \%$ in the UK. These authors considered that long-term exposure to high levels of $\mathrm{PM}_{2.5}$ is a significant co-factor that influences the severity of COVID-19 outcomes and increases the risk of mortality from SARS-CoV-2 [37].

However, the scope of these significant associations should be moderated because the health outcome considered, whether the number of cases of COVID-19 or the number of deaths, was measured during the first 2-3 months of the onset pandemic. It is a short period, during which the spread of the pandemic was mainly limited to parts of the countries, particularly because of the adoption of containment and physical distancing measures. We assume that the magnitude of the association between long-term exposure to $\mathrm{PM}_{2.5}$, and the COVID-19 mortality rate would tend to decrease or even disappear as the spread of the pandemic progresses and affects multiple geographic areas across the entire country. Our study has shown how this association is sensitive to the spread of the pandemic over different periods. Indeed, in the case of France, with COVID-19 mortality data as of 1 November 2020 a $1 \mu \mathrm{g} / \mathrm{m}^{3}$ increase in PM $\mathrm{PM}_{2.5}$ corresponded to a $15 \%$ increase in COVID-19 mortality rate, while with the data from the month of December, this association was no longer significant with the same variables. This result suggests that the association was not sustainable in the long term, and it might only represent a snapshot of the spread of the pandemic in the country [41].

\section{Association between COVID-19 mortality rate and temperature}

Several recent studies have analysed the influence of air quality on the spread of the COVID-19 pandemic and the resulting deaths [14, 18, $19,20,42-44]$. Most of the results of these studies suggest that temperature could play an important role in the spread of the COVID-19

Page $12 / 18$ 
pandemic, as in the case of the influenza epidemic. On the contrary, other studies have not found statistically significant associations between the incidence and/or mortality due to COVID-19 and temperature [43].

Our results highlight the existence of these two seemingly contradictory situations during the analysis period. In fact, until 1 October 2020 the COVID-19 mortality rate was not statistically associated with temperature. However, from 1 November, the association became significant.

Over the study period, the multiplication coefficient of the association between mortality rate and temperature was less than 1 , suggesting an inverse relationship between the two. However, this inverse relationship was statistically significant only with regards to the readings on 1 November, 1 December, and 31 December.

Thus, a degree of increase in the average temperature was associated with a 9.6\% decrease in the COVID-19 mortality rate on 1 November, $13.0 \%$ a month later on 1 December, and then $14.1 \%$ as of 31 December 2020 . These results could be explained by the fact that the COVID-19 mortality rate increased more sharply in the departments where low temperatures were more frequent than in the departments of the West and South-West of France.

Qi et al. [42] showed that both temperature and humidity are negatively associated with COVID-19.

In France, the emergence of the second wave coincided with the gradual drop in temperatures, notwithstanding the supposed relaxation of certain social distancing gestures, and tends to reinforce the hypothesis of a significant effect of temperature on the incidence and death rate of COVID-19. Our results are consistent with those found in China [18] and Spain in the Barcelona region [19]. Tobias and Molina [19] found that a $1^{\circ} \mathrm{C}$ increase in maximum temperature reduced the incidence rate by $7.5 \%$ on the same day. Holtman et al. [20] found that ambient temperature plays a significant role in the spread of COVID-19 by promoting the survival of the virus in the environment when temperatures are low. Wang et al. [14] reported that low temperature and low humidity significantly contributed to the transmission and survival of coronaviruses. For these authors, 'strict public health strategies should be continued when temperature drops in most parts of the country so as to prevent reversal of the epidemic'. Coker et al. [15] found a negative association between temperature and COVID-19 mortality. In contrast, Sobral et al. [43] found no significant correlation between the COVID-19 mortality rates and temperature.

Finally, our study confirms and extends the results of previous studies concerning the impact of environmental factors on the COVID-19 mortality rate.

The long observation period of cumulative deaths due to the COVID-19, from March to December 2020, has indeed made it possible to highlight the influence of the duration and extent of the spread of the COVID-19 on most of the results of previous studies. Typically, the significant positive association between the COVID-19 mortality rate and long-term exposure to $\mathrm{PM}_{2.5}$ did not cease until 1 December 2020, while the relationship between the mean annual temperature and COVID-19 mortality rate did not appear until 1 November 2020. This last period coincided with what many specialists called the second wave of the pandemic, which was observed in several European countries (such as the UK, Germany, Spain, Belgium, Portugal, and Italy). This second wave gained momentum as the temperature gradually dropped, resulting in more deaths than the first wave. Future research with data from other countries is needed to evaluate the consistency of the relationships observed in France between COVID-19 mortality and environmental factors. Likewise, the results of our study encourage us to pursue investigations with individual data when they are available and publicly accessible.

\section{Limitations}

This study presents some inherent limitations to the ecological analysis, and our findings should be interpreted with caution due to some biases related to ecological data. First, within departments, long-term exposure to PM 2.5 and COVID-19 deaths could vary from place to place, masking the heterogeneity within them. Second, the lack of individual data on COVID-19 deaths did not allow investigation of the precise long-term impact. In addition, the cross-sectional design of this study and the constraint of available data could also constitute limitations, as it is difficult to predict the evolution of the associations observed with time and the duration of the coronavirus pandemic itself. Furthermore, the COVID-19 pandemic is ongoing; hence, the study findings could change. Hence, the study results these should be considered intermediate results.

\section{Conclusions}


This study found significant associations between the COVID-19 mortality rate, long-term exposure to $\mathrm{PM}_{2.5}$, and temperature. Due to the health implications of well-documented air pollution, it could play a role as a co-factor in COVID-19 mortality through induced comorbidities. However, the influence of these associations tended to decrease with the evolution of the pandemic and massive spread of the disease across the country. Our results suggest that long-term exposure to $\mathrm{PM}_{2.5}$, even at concentrations lower than those set by the EU guidelines set $\left(25 \mu \mathrm{g} / \mathrm{m}^{3}\right)$, has a severe impact on the state of health of the population; it has a strong association with the COVID-19 mortality rate. Public authorities should implement effective strategies to improve air quality to at least approach the limits set by the WHO at $10 \mu \mathrm{g} / \mathrm{m}^{3}$. The analysis at the sub-national level makes it possible to identify the determinants of geographic inequalities in COVID-19 mortality rates and help policymakers, clinicians, and public health practitioners understand the spatial distribution of the pandemic and adapt intervention strategies for current or future pandemics.

\section{Declarations}

\section{Ethics approval and consent to participate}

This study used aggregated data from government and public sources that are openly available. It did not need ethical approval in this case.

\section{Consent for publication}

Not applicable

\section{Availability of data and materials}

We used publicly available data and have referenced the sources in the paper.

\section{Declaration of competing interests}

We declare no competing interests.

\section{Funding source}

This study had no funding for design, data collection, data analysis, data interpretation, or writing of the manuscript. A part of the contribution of Dr Omrani was supported by the Luxembourg National Research Fund under the programme COVID-19 Fast-Track (grant agreement No. COVID-19/2020-1/14718588/COVID-transmission).

\section{Authors' contributions}

AT: Conceptualization, Methodology, Formal analysis, Data curation, Writing- original draft, review \& editing, NL: Data curation, Formal analysis, Writing- review \& editing. HO: Software, Data curation, Writing-review \& editing. GDL: Data curation, Software, Writing- review \& editing. KL: Data curation, Writing- review \& editing. All authors reviewed and approved the final manuscript.

\section{Acknowledgements}

We thank the editorial team and anonymous referees for helpful comments. So, the views expressed herein are of the authors. We thank Editage for editorial assistance.

\section{Supplementary Material}

Supplementary material related to this article can be found online.

\section{References}

1. WHO Europe. Rolling updates on coronavirus disease, 2020 [accessed on May 15, 2020]. Available from: https://www.who.int/emergencies/diseases/novel-coronavirus-2019/events-as-they-happen

2. Pope CA, Burnett RT, Thurston GD, et al. Cardiovascular mortality anf long-term exposure to particulate air pollution: epidemiological evidence of general pathophysiological pathways of disease. Circulation, 2004, 109 (1), 71-77. 
3. Hajat A, Allison M, Diez-Roux AV, Jenny NS, Jorgensen NW, Szpiro AA et al. Long-term exposure to air pollution and markers of inflammation, coagulation, and endothelial activation: a repeat-measures analysis in the Multi-Ethnic Study of Atherosclerosis (MESA). Epidemiology. 2015; 26(3): 310-20. [PMC free article] [PubMed] [Google Scholar].

4. Beelen R, Raaschou-Nielson O, Stafoggia M, Andersen ZJ, Weinmayr G, Hoffmann B, et al. Effects of long-term exposure to air pollution on natural-cause mortality : An analysis of 22 European cohorts within in the multicentre ESCAPE project. Lancet 383 (9919):785-795 (2014). Doi:10.1016/S0140-6736(13)62158-3.

5. European Environment Agency (EEA). Air quality in Europe- 2018 Report. EEA Report No. 12/2018. 88 pages.

6. Tonne C, Halonen JI, Beevers SD, Dajnak D, Gulliver J, Kelly FJ et al. Long-term traffic air and noise pollution in relation to mortality and hospital readmission among myocardial infarction survivors. Int J Hyg Environ Health. 2016; 219(1):72-8. [PubMed].

7. EEA. Air Quality in Europe Report Number 10/2019. [accessed on 22 May 2020]; Available online: https://www.eea.europa.eu/publications/air-quality-in-europe-2019.

8. Wu X, Nethery RC, Sabath B.M, Braun D, Dominici F. Exposure to air pollution and COVID-19 mortality in the United States: A nationwide cross-sectional study. medRxiv. 2020 Apr 7 and Preprint, 2020.04.05.20054502. doi: 10.1101/2020.04.05.20054502.

9. Beelen R, Stafoggia M, Raaschou-Nielsen O, Andersen ZJ, Xun WW, Katsouyanni K et al. Long-term exposure to air pollution and cardiovascular mortality: an analysis of 22 European cohorts. Epidemiology. 2014; 25(3):368-78. [PubMed] [Google Scholar].

10. Liu C, Chen R, Sera F, Vicedo-Cabrera AM, Guo Y, Tong S, et al. Ambient particulate air pollution and daily mortality in 652 cities. N. Engl. J. Med. 2019 and Doi:101056/NEJMoa1817364, 381 (8):705-715.

11. Di Q, Wang Y, Zanobetti A, Wang Y, Koutrakis P, Choirat C, Dominici F, and Schwartz J D. Air pollution and mortality in the Medicare population. N. Engl. J. Med. 2017 and 10.1056/NEJMoa1702747, 376: 2513-2522. Doi:.

12. Pappin AJ, Christidis T, Pinault LL, Crouse DL, Brook JR, Erickson A, et al. Examining the shape of the association between low levels of fine particulate matter and mortality across three cycles of the Canadian Census Health and Environment Cohort. $\mathrm{E}$.

13. Wu X, Nethery RC, Sabath MB, Braun D, Dominici F. Exposure to Air Pollution and COVID-19 Mortality in the United States. [(accessed on 22 May 2020)]. Available online: https://projects.iq.harvard.edu/covid-pm.

14. Wang B, Chen H, Chan Y-L, and Oliver BG. Is there an association between the level of ambient air pollution and COVID-19? Am J Physiol Lung Cell Mol Physiol 319: L416-L421, 2020. First published July 22, 2020 and 10.1152/ajplung.00244.2020.

15. Coker ES, Cavalli L, Fabrizi E, Guastella G, Lippo E, Parisi ML, Pontarollo N, Rizzati M, Varacca A, Vergalli S. The effects of air pollution on COVID-19 related mortality in northern Italy. Environmental and Resource Economics. https://doi.org/10.1007/.

16. Zoran M, Savastru RS, Savastru DM, Tautan MN. Assessing the relationship between surface levels of PM2.5 and PM10 particulate matter impact on COVID-19 in Milan, Italy. Science of the Total Environment 738 (2020) 139825. https://doi.org/10.1016/j.scito.

17. Comunian S, Dongo D, Milani C, and Palestini P. Air pollution and COVID-19: The role of particulate matter in the spread and increase of COVID-19's morbidity and mortality. Int. J. Environ. Res. Public Health 2020, 17, 4487 and Pu, doi:10.3390/ijerph17124487.

18. Shi P, Dong Y, Yan H, Zhao C, Li X, Liu W, He M, Tang S, Xi S. Impact of temperature on the dynamics of the COVID-19 outbreak in China. Science of the Total Environment 728 (2020) 138890. https://doi.org/10.1016/j.scitotenv.2020.138890. Available online 23 April 2020.

19. Tobias A and Molina T. Is temperature reducing the transmission of COVID-19? Environmental Research, 2020, 186, 109553.

20. Holtmann M, Jones M, Shah A, and Holtmann G. Low ambient temperatures are associated with more rapid spread of COVID-19 in the early phase of the endemic. Environment Research 186 (2020) 109625. https://doi.org/10.1016/j.envres.2020.109625, available online 06 May 2020.

21. Omrani, H., Modroiu, M., Lenzi, J., Omrani, B., Said, Z., Suhrcke, M., \& Parmentier, B. COVID-19 in Europe: Dataset at a sub-national level. Data in Brief, 106939. (2021).

22. Lelieveld J, Klingmüller, Pozzer A, Pöschl U, Fnais M, Daiber A, Münzel T. Cardiovascular disease burden from ambient air pollution in Europe reassessed using novel hazard ratio functions. European Heart Journal (2019) 40, 1590-1596. Doi:10.1093/eurheartj/ehz135.

23. Coccia M. Factors determining the diffusion of COVID-19 and suggested strategy to prevent future accelerated viral infectivity similar to COVID. Science of the Total Environment 729 (2020) 138474. https:doi.org/10.1016/j.scitotenv.2020.138474. Available online 20 A.

24. Conticini E, Frediani B, Caro D. Can Atmospheric Pollution Be Considered a Co-Factor in Extremely High Level of SARS-CoV-2 Lethality in Northern Italy? [(accessed on 20 May 2020)];Environ. Pollut. 2020 doi: 10.1016/j.envpol.2020.114465. Available online: https://www.ncbi.nlm.nih.gov/pmc/. 
25. Wu X, Nethery RC, Sabath B.M, Braun D, Dominici F. Exposure to air pollution and COVID-19 mortality in the United States: A nationwide cross-sectional study. medRxiv. 2020 Apr 7;2020.04.05.20054502. doi: 10.1101/2020.04.05.20054502. Preprint.

26. Villeneuve PJ and Goldberg MS. Methodological Considerations for Epidemiological Studies of Air Pollution and the SARS and COVID-19 Coronavirus Outbreaks. Environmental Health Perspectives, 128, 9, September 2020. https://doi.org/10.1289/EHP7411.

27. Barron E, Bakhai, C, Kar P, Weaver A, Bradley D, Ismail H, Knighton P, Holman N, Khunti K, Sattar N, Wareham N J, Young B, Valabhji J. Association of type 1 and type 2 diabetes with COVID-19-related mortality in Englan: a whole-population study. Lancet Diabetes Endocrinal 2020; 8:813-22. Published Online August 13, 2020. https://doi.org/10.16/S2213-8587(20)30272-2.

28. Apicella M, Campopiano MC, Mantuano M, Mazoni L, Coppelli A, Del Prato S. COVID-19 in people with diabetes: understanding the reasons for worse outcomes. Lancet Diabetes Endocrinol. 2020 Jul 17;S2213-8587(20)30238-2. doi: 10.1016/S22138587(20)30238-2. Online ahead of print.

29. Liu S and Li M. Ambient air pollutants and their effect on COVID-19 mortality in the United States of America. Rev Panam Salud Publica. 2020; 44:e159. https://doi.org/10.26633/RPSP.2020.159.

30. Allison PD. 2005. Fixed effects regression methods for longitudinal data. SAS. Cary, NC: SAS Institute Inc.

31. Wu X, Nethery RC, Sabath MB, Braun D, Dominici F. Air pollution and COVID-19 mortality in the United States: Strengths and limitations of an ecological regression analysis. Sci. Adv. 2020; 6 (45): eabd4049, 4 November 2020. Doi:10.1126/sciadv.abd4049.

32. Andrée BPJ. Incidence of COVID-19 and connections with air pollution exposure: Evidence from the Netherlands. World Bank Group, Policy Research Working Paper 9221, April 2020, 27 pages.

33. Sun F, Matthews SA, Yang TC, Hu MH. A spatial analysis of the COVID-19 period prevalence in U.S. counties through June 28, 2020: where geography matters? Annals of Epidemiology, https://doi.org/10.1016/j.annepidem.2020.07.014.

34. Pansini R., Fornacca D. Initial evidence of higher morbidity and mortality due to SARS-CoV-2 in regions with lower air quality. [(accessed on 22 May 2020)];medRxiv. 2020 Available online: https://www.medrxiv.org/content/10.1101/2020.04.04.20053595v2. DOI.

35. Wang Y, Di Q. Modifiable areal unit problem and environmental factors of COVID-19 outbreak. Science of the Total Environment 740 (2020) 139984. https://doi.org/10.1016/j.scitotenv.2020.139984. Available online 6 June 2020.

36. Setti L, Passarini F, de Gennaro G, Di Gilio A, Palmisani J, Buono P, Fornari G, Perrone MG, Piazzalunga A, Barbieri P, et al. Evaluation of the Potential Relationship between Particulate Matter (PM) Pollution and COVID-19 Infection Spread in Italy. [(accessed on 22 May 2020)]; Available online: http://www.simaonlus.it/wpsima/wp-content/uploads/2020/03/COVID_19_position-paper_ENG.

37. Pozzer A, Dominici F, Haines A, Witt C, Münzel T, and Lelieveld J. Regional and global contributions of air pollution to risk of death from COVID-19. Cardiovascular Research (2020) 116, 2247-2253. Doi :10.1093/cvr/cvaa288. Online publish-ahead-of-print 26 October 2020.

38. Sharma AK, Balyan P. Air pollution and COVID-19: Is the connect worth its weight?. Indian J Public Health [serial online] 2020 [cited 2021 Apr 8];64, Suppl S2:132-4. Available from: https://www.ijph.in/text.asp?2020/64/6/132/285597.

39. Wang Y, Shi L, Lee $\mathrm{M}$, et al. Long-term exposure to $\mathrm{PM}_{2.5}$ and mortality among older adults in the southeastern US. Epidemiology 2017, 28 (2), 207.

40. Cole M A, Ozgen C, Strobl E. Air pollution exposure and COVID-19. IZA DP No. 13367, June 2020.28 pages.

41. Office for National Statistics (ONS). Coronavirus (COVID-19) related mortality rates and the effects of air pollution in England. Research report examining the potential relationship between long-term air pollution exposure and coronavirus (COVID-19) mortality rates. Last revised 13 August 2020. [Accessed 12 October 2020] Available online: https://www.ons.gouv.uk/.

42. Qi H, Xia S, Shi R., Runye S. et al. COVID-19 transmission in Mainland China is associated with temperature and humidity: A timeseries analysis. Sci Total Environ. 2020 Aug 1;728:138778. doi: 10.1016/j.scitotenv.2020.138778. Epub 2020 Apr 19.

43. Sobral MFF, Duarte GB, Sobral AIGP, Marinho MLM, Melo AS. Association between climate variables and global transmission of SARS-CoV-2. Science of the Total Environment 729 (2020) 138997. https://doi.org/10.1016/j.scitotenv.2020.138997.

44. Yuan S, Jiang SC, and Li ZL (2020). Do humidity and temperature impact the spread of the novel coronavirus? Front. Public Health 8:240. Doi:10.3389/fpubh.2020.00240.

\section{Figures}




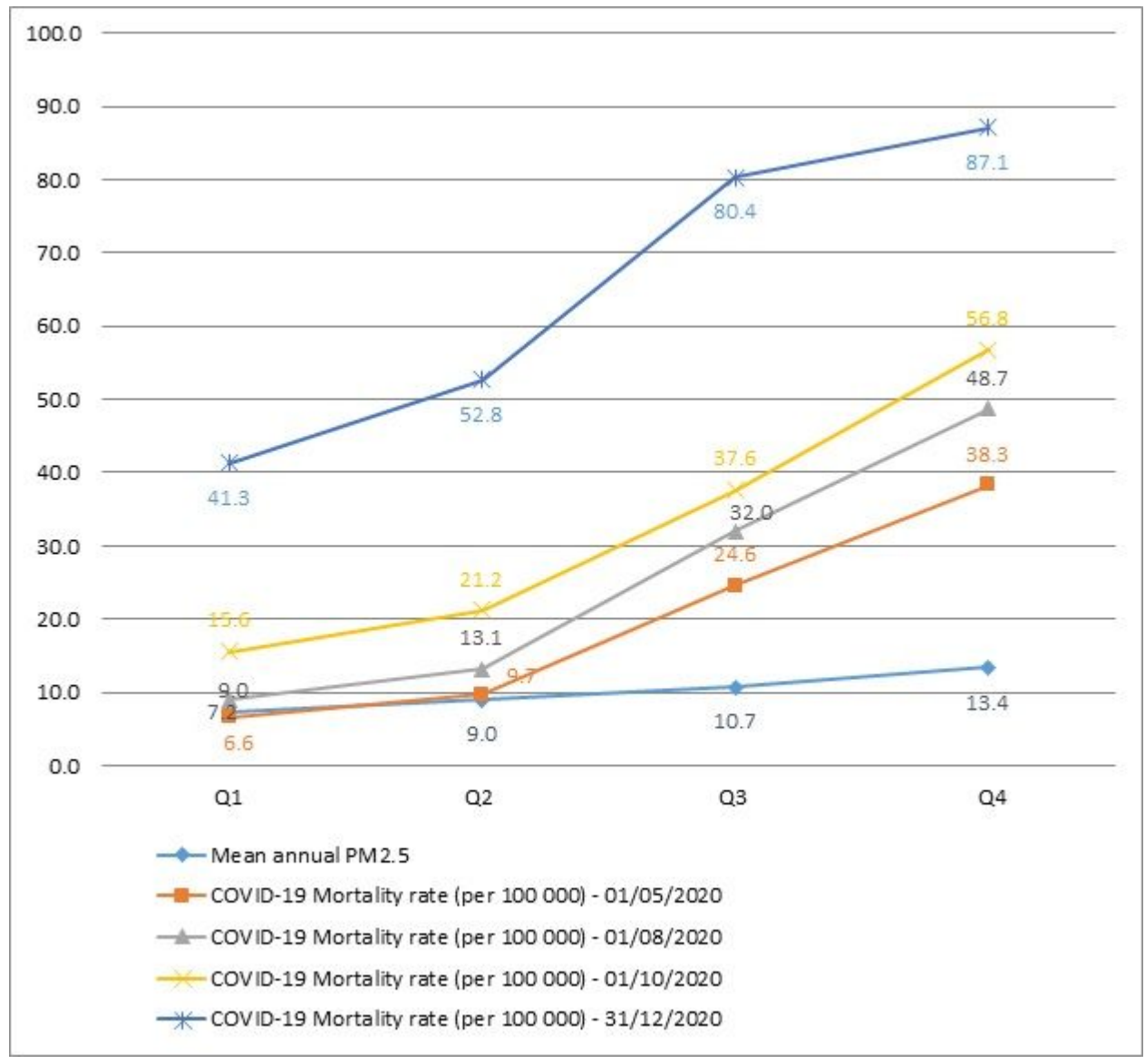

Figure 1

Distribution of the COVID-19 mortality rate and long-term PM2.5 exposure per quartile 


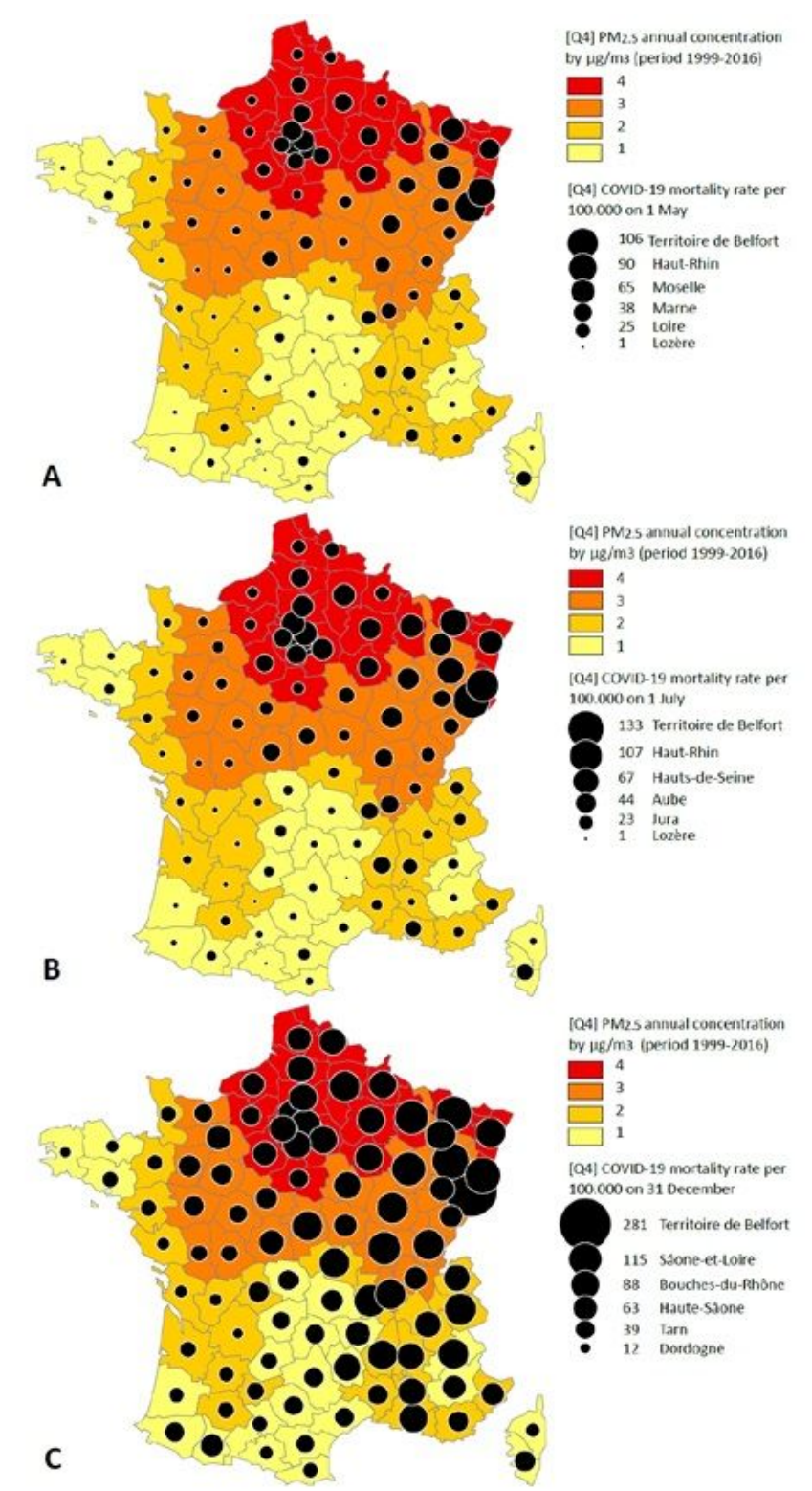

Figure 2

Map 1: Geographical distribution of the COVID-19 mortality rate depending to quartile of PM2.5 concentration level by department on 1 May 2020 (a), 1 July (b), and 31December 2020 (c)

\section{Supplementary Files}

This is a list of supplementary files associated with this preprint. Click to download.

- COVID19Francesuplementaryappendixv07May2021.docx 